\title{
Técnica operatoria de apendicetomía e infección del sitio operatorio. Hospital María Auxiliadora. Octubre-diciembre, 2018
}

\author{
Ursula Alexandra Sandoval Gionti 1,a, Ysabel Lozano Rodas* 1,2,b,c; Enver Vantroi Palacios Ordoñez 1,2,b,c; Jorge Kohatsu \\ Yshida $1,3, \mathrm{~b}, \mathrm{~d}$
}

RESUMEN

Objetivo: Determinar la incidencia y los factores asociados a infección de sitio operatorio en pacientes apendicectomizados en el Servicio de Cirugía General del Hospital María Auxiliadora durante el periodo octubre-diciembre 2018.

Materiales y métodos: Estudio transversal, tipo serie de casos comparativo en una cohorte histórica de pacientes apendicectomizados durante el periodo de estudio. Los datos se obtuvieron de historias clínicas de pacientes intervenidos mediante tres técnicas operatorias (apendicectomía transversa, transumbilical y laparoscópica). Se determinó la presencia de infección de sitio operatorio, características clínico-demográficas y factores asociados al desarrollo de esta complicación.

Resultados: El 20 \% de pacientes apendicectomizados presentaron infección del sitio operatorio. La mayoría de los casos fueron posteriores a la técnica operatoria transversa (56\%) (p: 0,03) y en los pacientes con apendicitis complicada (54\%) $(<0,001)$, que requirieron un mayor tiempo de estancia hospitalaria (4 días) (RIQ: $2-6)(p<0,01)$. En el análisis de regresión múltiple se evidenció asociación entre la técnica operatoria (apendicectomía transumbilical y transversa) e infección de sitio operatorio [IRR: 3,72 IC95 \% $(1,58-8,75)$ y 2,63 IC95 \% $(1,27-5,44)]$, y apendicitis complicada [IRR: 5,56 IC95 \% $(2,32$ 13,35)].

Conclusiones: La infección de sitio operatorio es una complicación frecuente de la apendicectomía y se asocia al tipo de técnica operatoria empleada y a los cuadros de apendicitis complicada.

Palabras clave: Apendicectomía; Infección; Herida quirúrgica (Fuente: DeCS BIREME).

\section{Appendectomy surgical technique and surgical site infection. Hospital María Auxiliadora. October - December 2018}

\section{ABSTRACT}

Objective: To determine the incidence and factors associated with surgical site infection in patients who underwent an appendectomy in the General Surgery Unit of the Hospital María Auxiliadora from October to December 2018.

Materials and methods: A cross-sectional comparative-case study was conducted in a historical cohort of patients who underwent an appendectomy during the study period. Data was obtained from medical records of patients who underwent surgery using three surgical techniques (transverse, transumbilical and laparoscopic appendectomy). Surgical site infection, clinical demographic characteristics and factors associated with the development of this complication were determined.

Results: Twenty percent (20\%) of the patients who underwent an appendectomy presented surgical site infection, mostly those operated with the transverse surgical technique (56\%) ( $p=0.03)$ and those with complicated appendicitis (54\%) $(p<0.001)$, who required a longer hospital stay of 4 days (IQR: $2-6)(p<0.01)$. A multiple regression analysis showed an association between surgical technique (transumbilical and transverse appendectomy) and surgical site infection [IRR: 3.72; $95 \% \mathrm{Cl}(1.58-8.75)$ and $2.6395 \% \mathrm{Cl}$ (1.27-5.44)] and development of complicated appendicitis [IRR: $5.5695 \% \mathrm{Cl}$ (2.32-13.35). Conclusions: Surgical site infection is a frequent complication of appendectomy and is associated with the surgical technique and complicated appendicitis.

Keywords: Appendectomy; Infection; Surgical wound (Source: MeSH NLM).

\footnotetext{
1. Universidad Científica del Sur. Lima, Perú.

2. Hospital María Auxiliadora, Servicio de Medicina Interna. Lima, Perú.

3. Hospital María Auxiliadora, Servicio de Cirugía General. Lima, Perú.

a. Estudiante.

b. Docente.

c. Médico Internista.

d. Médico Cirujano.

*Autor corresponsal
} 


\section{INTRODUCCIÓN}

La Organización Mundial de la Salud define infección de sitio operatorio (ISO) a aquella infección que se presenta dentro de los 30 días posteriores a una cirugía y que puede involucrar la piel, el tejido celular subcutáneo, músculos, fascias, órganos y espacios ${ }^{(1,2)}$. Por tal motivo, la ISO es una complicación potencialmente asociada a cualquier tipo de procedimiento quirúrgico.

Según la OMS, en el año 2014 en Estados Unidos, hubieron 20916 infecciones de sitio operatorio de 2417 933 procedimientos quirúrgicos. En Francia, se estimó que el $3 \%$ de procedimientos quirúrgicos resultantes en infecciones generan un costo anual aproximado de 58 millones de euros, sin mencionar que los pacientes con ISO presentaron un aumento del riesgo de mortalidad y un incremento del tiempo de estancia hospitalaria. Estudios previos en países desarrollados reportan incidencias de ISO en pacientes apendicectomizados entre 2,1 a $6 \%{ }^{(3)}$. En América Latina, se ha reportado incidencias desde $15 \%$ hasta $41,9 \%(4,5)$.

El procedimiento quirúrgico más frecuente en las emergencias es la apendicectomía ${ }^{(6)}$, y la apendicectomía abierta es la única técnica quirúrgica aceptada por varios años. Sin embargo, con el pasar del tiempo nuevas técnicas operatorias han ido apareciendo con la finalidad de reducir las potenciales complicaciones postoperatorias como es la ISO, tal como se evidencia en estudios publicados en Qatar donde se reportan incidencias de ISO de 1,7\% para apendicectomía laparoscópica y 20,6\% para cirugía abierta ${ }^{(7)}$; en España, un $14 \%$ para apendicectomía laparoscópica y $13,4 \%$ para cirugía abierta ${ }^{(8)}$; mientras que en Corea no se encontraron diferencias significativas entre ambas técnicas quirúrgicas ${ }^{(9)}$.

A pesar de que las ISO son infecciones prevenibles, se encuentran entre los cuadros nosocomiales más frecuentes y representan una carga significativa en términos de morbimortalidad y costos adicionales ${ }^{\left({ }^{10}\right)}$ para los sistemas de salud y pagadores de servicios. De igual manera, la apendicitis aguda se presenta en personas pertenecientes a la población económicamente activa, de forma que la ISO retardaría la reincorporación a su actividad habitual.

El Hospital María Auxiliadora, dependencia del Ministerio de Salud, es una institución asistencial de tercer nivel que funciona como centro hospitalario de referencia en el Cono Sur de Lima. La cirugía más frecuente es la apendicectomía, que se realiza mediante una de las siguientes técnicas: laparoscópica o abierta, de incisión transversa y de incisión transumbilical. Durante el año 2017, en el Servicio de Emergencia de Cirugía General, se realizaron 1284 cirugías, de las cuales 839 fueron apendicectomías (11). En nuestro país hay pocos estudios sobre la incidencia de ISO postapendicectomía de acuerdo a la técnica operatoria empleada y factores asociados a su desarrollo, por lo que el presente trabajo permitirá conocer estos datos epidemiológicos importantes para así poder optar por la técnica con menores complicaciones.

\section{MATERIALES Y MÉTODOS}

\section{Diseño y población de estudio}

Se realizó un estudio transversal tipo serie de casos comparativo en una cohorte histórica de pacientes que fueron sometidos a una intervención quirúrgica de apendicectomía en el Servicio de Cirugía General del Hospital María Auxiliadora durante el periodo octubrediciembre 2018. Se hizo un muestreo no probabilístico, por conveniencia, consecutivo de todas las historias clínicas de pacientes apendicectomizados de incisión transversa, transumbilical y apendicectomía laparoscópica. La muestra consistió en 150 historias clínicas con datos completos.

Entre los criterios de inclusión se consideraron a todos los pacientes apendicectomizados durante el periodo de estudio, mayores a 8 años de edad. Los criterios de exclusión fueron pacientes menores de 7 años 11 meses y 30 días pertenecientes al Servicio de Cirugía Pediátrica, pacientes apendicectomizados mediante la técnica de laparatomía exploratoria y los que ingresaron a sala de operaciones para realizar apendicectomía transversa, transumbilical o laparoscópica pero que tuvieron que ser convertidas a laparatomía.

\section{Variables y medición}

La información se obtuvo mediante la aplicación de una ficha de datos, con la cual se recolectó información de las historias clínicas. El instrumento explora las distintas variables de estudio. La variable dependiente es la infección de sitio operatorio, la cual se definió como infección de la incisión quirúrgica o cerca de ella, que se presentó dentro de los 30 días posteriores a la realización de la apendicectomía y que fue diagnosticada y registrada en la historia clínica por un médico cirujano. Las variables independientes son edad, sexo, índice de masa corporal (IMC =peso/talla ${ }^{2}$ ), comorbilidades, tiempo de enfermedad (en horas), tiempo operatorio (en minutos), diagnósticos preoperatorio y postoperatorio [apendicitis complicada (necrosada, perforada) y no complicada (sin alteraciones significativas, congestiva y flemonosa)], técnica operatoria y estancia hospitalaria.

\section{Análisis estadístico}

Se realizó un análisis descriptivo de las características basales de la población de estudio. Los datos cualitativos 
se presentan en forma de frecuencias absolutas y porcentajes, y los datos cuantitativos mediante medidas de tendencia central y de dispersión (media, mediana, desviación estándar y rango intercuartílico). Luego se realizó un análisis bivariado entre los tres grupos de pacientes (incisión transversa, transumbilical y laparoscópica). Las variables cualitativas (sexo, diabetes mellitus, técnica operatoria y diagnóstico postoperatorio) se analizaron mediante el test de Chi cuadrado o el test exacto de Fisher, y las cuantitativas (edad, IMC, nivel de hemoglobina, tiempo operatorio, tiempo de enfermedad, estancia hospitalaria) con pruebas paramétricas o no paramétricas de acuerdo a la distribución de la variable. Finalmente, se realizó un modelo de regresión GLM binomial log simple y múltiple, para el cálculo de la razón de incidencias (al ser la variable resultado ISO una complicación que aparecía tras la intervención, por lo tanto, se tiene la seguridad que el evento no estaba presente y por lo tanto era de aparición reciente, pudiendo hablar de casos incidentes), sus respectivos intervalos de confianza y para ajustar las covariables que se asociaron a la aparición de la ISO. Se consideró como nivel de significancia estadística $p<0,05$ y se utilizó el paquete estadístico Stata 15.

\section{RESULTADOS}

Se evaluaron 150 historias clínicas de pacientes apendicectomizados en el Servicio de Cirugía General del Hospital María Auxiliadora entre octubre - diciembre 2018, en las que se encontró una incidencia de $20 \%$ de ISO. El tiempo de enfermedad fue de 60 horas (RIQ: 18-47), la técnica operatoria más empleada fue la apendicectomía abierta en su modalidad transversa $(43,4 \%)$. La mediana de tiempo operatorio fue de 60 minutos (RIQ: 42-70) y se observó que el diagnóstico preoperatorio y postoperatorio más frecuente fue apendicitis aguda complicada (62,7 \%, $54 \%$ respectivamente). Los pacientes tuvieron una mediana de estancia hospitalaria de 2 días (RIQ: 1-4). Pocos pacientes presentaron comorbilidades, entre ellas, diabetes mellitus (3,3\% de los casos), así mismo se evidenció un IMC de 26,4 \pm 4,7 (Tabla 1).

Tabla 1. Características de los pacientes apendicectomizados. Hospital María Auxiliadora. Octubre-diciembre, 2018

\begin{tabular}{|c|c|}
\hline Características & $\mathbf{N}(\%)$ \\
\hline Edad (años) ${ }^{*}$ & $25[16-40]$ \\
\hline \multicolumn{2}{|l|}{ Sexo } \\
\hline Femenino & $65(43)$ \\
\hline Masculino & $85(56,4)$ \\
\hline \multicolumn{2}{|l|}{ Técnica operatoria } \\
\hline Laparoscopía & $66(44,0)$ \\
\hline Transumbilical & $19(12,7)$ \\
\hline Transversa & $65(43,4)$ \\
\hline $\mathrm{IMC}^{* *}$ & $26,4( \pm 4,7)$ \\
\hline \multicolumn{2}{|l|}{ Diabetes mellitus } \\
\hline Sí & $5(3,3)$ \\
\hline \multicolumn{2}{|l|}{ Hepatopatía crónica } \\
\hline Sí & $0(0)$ \\
\hline \multicolumn{2}{|l|}{ Inmunosupresión } \\
\hline Sí & $0(0)$ \\
\hline Hemoglobina (gr/dl) ${ }^{*}$ & $14[12,8-14,7]$ \\
\hline Tiempo de enfermedad (horas) ${ }^{*}$ & $26,5[18-47]$ \\
\hline Tiempo operatorio (minutos)* & $60[42-70]$ \\
\hline \multicolumn{2}{|l|}{ Diagnóstico preoperatorio } \\
\hline Apendicitis aguda no complicada & $48(32)$ \\
\hline Apendicitis aguda complicada & $94(62,7)$ \\
\hline Abdomen agudo quirúrgico & $8(5,3)$ \\
\hline
\end{tabular}




\begin{tabular}{|c|c|}
\hline Características & $\mathbf{N}(\%)$ \\
\hline \multicolumn{2}{|l|}{ Diagnóstico postoperatorio } \\
\hline Apendicitis aguda no complicada & $69(46)$ \\
\hline Apendicitis aguda complicada & $81(54)$ \\
\hline Estancia hospitalaria (días) ${ }^{*}$ & $2[1-4]$ \\
\hline \multicolumn{2}{|l|}{ ISO } \\
\hline No & $120(80)$ \\
\hline Sí & $30(20)$ \\
\hline \multicolumn{2}{|l|}{ Grado de ISO } \\
\hline Incisional superficial & $23(76,7)$ \\
\hline Incisional profunda & $1(3,3)$ \\
\hline Órgano y espacio & $6(20)$ \\
\hline
\end{tabular}

*Mediana (rango intercuartílico); **Media \pm DS; IMC: Índice de masa corporal; PF: Peritonitis focal; ISO: Infección de sitio operatorio.

Los pacientes que presentaron ISO habían sido sometidos a una apendicetomía abierta, en la mayoría de los casos $(p=0,03)$. El diagnóstico postoperatorio predominante es la apendicitis aguda complicada $(<0,01)$ (Figura 1). La mediana de estancia hospitalaria fue mayor en los pacientes que presentaron ISO $(p<0,01)$. No hubo diferencia estadísticamente significativa entre los grupos en cuanto a edad, sexo, tiempo operatorio, tiempo de enfermedad e IMC (Tabla 2).

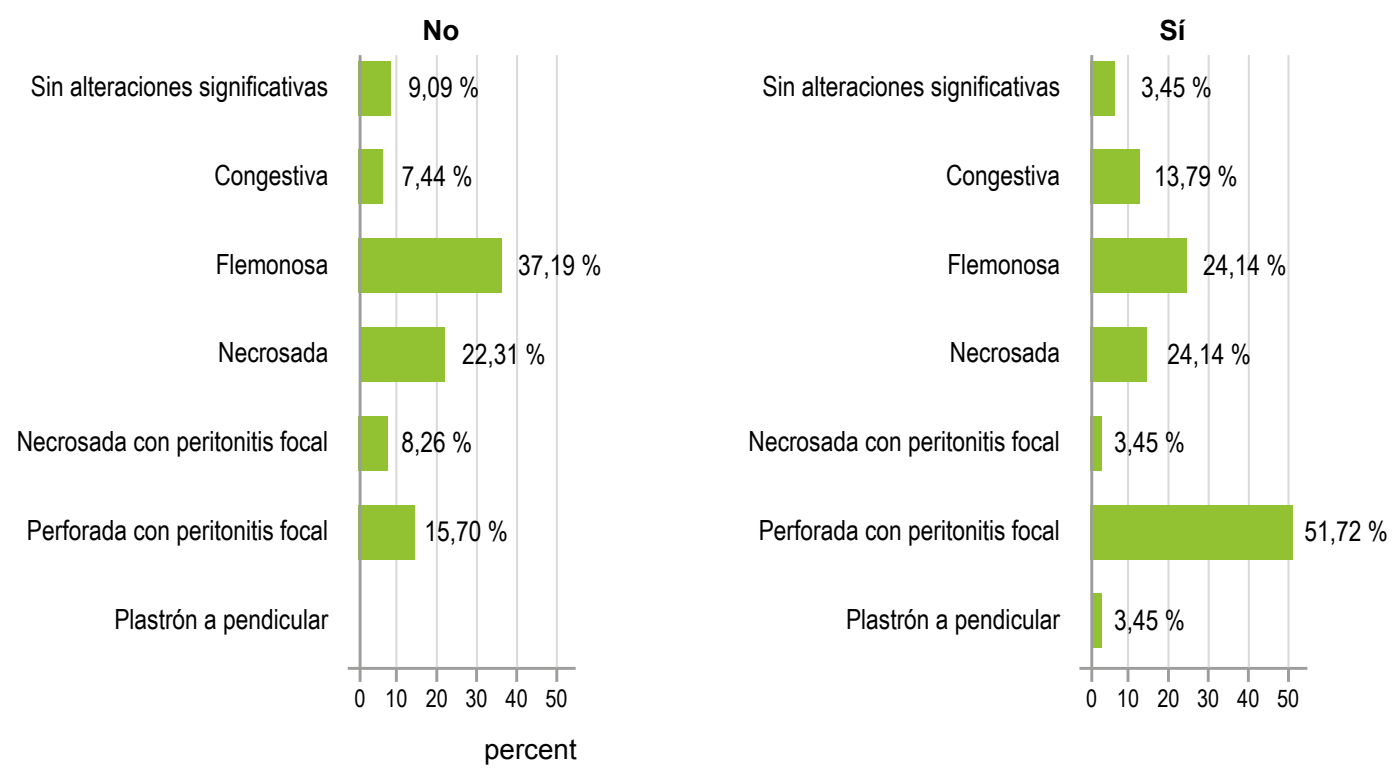

Figura 1. Pacientes apendicectomizados con ISO según diagnóstico postoperatorio. Hospital María Auxiliadora. Octubre-diciembre, 2018

En la regresión simple se encontró que la técnica operatoria está asociada a la infección de sitio operatorio, siendo un factor de riesgo (transumbilical: RR: 2,98, IC95 \% 1,14-7,80 y transversa RR: 2,47, IC95 \% 1,10-5,55). Asimismo, se observó que el diagnóstico postoperatorio de apendicitis aguda complicada es un factor asociado al desarrollo de ISO (RR: 4,26, IC95 \% 1,72-10,53). La asociación entre infección del sitio operatorio y técnica operatoria 
(transumbilical aRR: 5,22, IC95 \% 2,34-11,66 y transversa aRR: 2,76 IC95 \% 1,27-6,04) se mantuvo luego de ser ajustada por diagnóstico postoperatorio. El desarrollo de infección de sitio operatorio es un factor de riesgo para mayor estancia hospitalaria (Figura 2 y tabla 3 ).

Tabla 2. Factores asociados a ISO en pacientes apendicectomizados en análisis bivariado

\begin{tabular}{|c|c|c|c|}
\hline \multirow[t]{3}{*}{ Variable } & \multicolumn{2}{|c|}{ Infección de sitio operatorio } & \multirow[t]{3}{*}{$\mathbf{P}$} \\
\hline & Sí (30) & No (120) & \\
\hline & n (\%) & n (\%) & \\
\hline & $27,5(15-48)$ & $24,5(16-39,5)$ & 0,26 \\
\hline \multicolumn{4}{|l|}{ Sexo } \\
\hline Femenino & $13(43,3)$ & $52(43,3)$ & $>0,99$ \\
\hline Masculino & $17(56,7)$ & $68(56,7)$ & \\
\hline \multicolumn{4}{|l|}{ Técnica operatoria } \\
\hline Laparoscópica & $7(23,3)$ & $59(49,2)$ & 0.03 \\
\hline Transumbilical & $6(20,0)$ & $13(10,8)$ & \\
\hline Transversa & $17(56,7)$ & $48(40,0)$ & \\
\hline $\operatorname{IMC}\left(\mathbf{k g} / \mathrm{m}^{2}\right)^{\star *}$ & $26,6 \pm 4,9$ & $26,3 \pm 4,6$ & 0,74 \\
\hline Hemoglobina (gr/dl)* & $14,1(13,2-14,7)$ & $13,7(12,6-14,7)$ & 0,38 \\
\hline \multicolumn{4}{|l|}{ Diabetes mellitus } \\
\hline Sí & $1(3,3)$ & $4(3,3)$ & $>0,99$ \\
\hline Tiempo de enfermedad (horas) ${ }^{*}$ & $31(19-52)$ & $26(17-44,5)$ & 0,17 \\
\hline Tiempo operatorio (minutos) ${ }^{*}$ & $57,5(42-65)$ & $60(42,5-73,5)$ & 0,3 \\
\hline \multicolumn{4}{|l|}{ Diagnóstico postoperatorio } \\
\hline Apendicitis aguda no complicada & $5(46)$ & $64(53,3)$ & $<0,01$ \\
\hline Apendicitis aguda complicada & $25(54)$ & $56(46,7)$ & \\
\hline Estancia hospitalaria (días)* & $4(2-6)$ & $2(1-4)$ & $<0,01$ \\
\hline
\end{tabular}

*Mediana (rango intercuartílico); **Media \pm DS; IMC: Índice de masa corporal

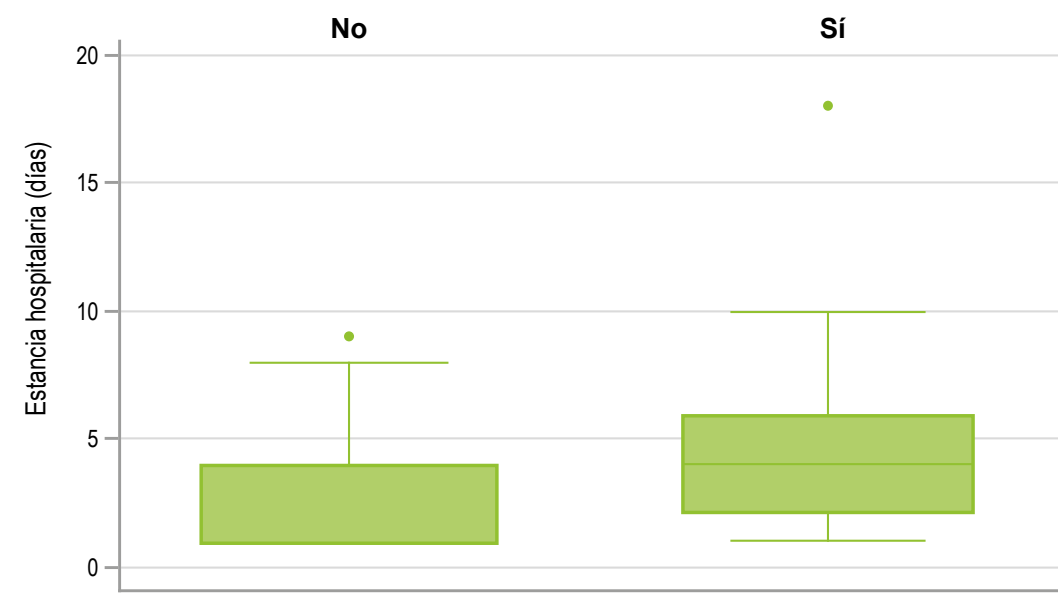

Figura 2. Pacientes apendicectomizados con ISO según estancia hospitalaria. Hospital María Auxiliadora. Octubre-diciembre, 2018 
Tabla 3. Factores asociados a infección de sitio operatorio en pacientes apendicectomizados. Hospital María Auxiliadora. Octubrediciembre, 2018

\begin{tabular}{|c|c|c|c|c|c|c|}
\hline \multirow[t]{2}{*}{ Características } & \multicolumn{3}{|c|}{ Análisis bivariado } & \multicolumn{3}{|c|}{ Regresión múltiple * } \\
\hline & IRR & IC $95 \%$ & $\mathbf{P}$ & IRR & IC $95 \%$ & $\mathbf{P}$ \\
\hline \multicolumn{7}{|l|}{ Sexo } \\
\hline Femenino & Ref. & & & & & \\
\hline Masculino & 1 & $0,52-1,91$ & $>0,99$ & & & \\
\hline Edad (años) * & 1,01 & $0,99-1,03$ & 0,15 & & & \\
\hline IMC $\left(\mathbf{k g} / \mathrm{m}^{2}\right)$ & 1,01 & $0,94-1,08$ & 0,74 & & & \\
\hline $\mathrm{Hb}$ (gr/dl) & 1,07 & $0,93-1,22$ & 0,37 & & & \\
\hline T. enfermedad (horas) & 1,01 & $0,99-1,02$ & 0,23 & & & \\
\hline T. operatorio (minutos) & 0,99 & $0,97-1,07$ & 0,23 & & & \\
\hline Estancia hospitalaria (días) & 1,18 & $1,11-1,26$ & $<0,01$ & & & \\
\hline \multicolumn{7}{|l|}{ Técnica operatoria } \\
\hline Laparoscópica & Ref. & & & Ref. & & \\
\hline Transumbilical & 2,98 & $1,14-7,80$ & 0,03 & 5,22 & $2,34-11,66$ & $<0,01$ \\
\hline Transversa & 2,47 & $1,10-5,55$ & 0,03 & 2,76 & $1,27-6,04$ & 0,01 \\
\hline \multicolumn{7}{|l|}{ Diagnóstico postoperatorio } \\
\hline Apendicitis aguda no complicada & Ref. & & & Ref. & & \\
\hline Apendicitis aguda complicada & 4,26 & $1,72-10,53$ & $<0,01$ & 5,56 & $2,32-13,35$ & $<0,01$ \\
\hline \multicolumn{7}{|l|}{ Diabetes mellitus } \\
\hline Sí & 1 & $0,17-5,98$ & $>0,99$ & & & \\
\hline No & Ref. & & & & & \\
\hline
\end{tabular}

*Ajustado por diagnostico postoperatorio; IRR: Incremento de riesgo relativo; IC: Intervalo de confianza; IMC: Índice de masa corporal.

\section{DISCUSIÓN}

El presente estudio encontró una incidencia de $20 \%$ de infección de sitio operatorio en los pacientes apendicectomizados en el Servicio de Cirugía General del Hospital María Auxiliadora, lo que evidencia que la técnica operatoria está asociada al desarrollo de infección de sitio operatorio siendo ésta un factor de riesgo.

Con relación a la incidencia de ISO en el Hospital María Auxiliadora, esta es ligeramente mayor a la reportada por Hernández JS ${ }^{(4)}$ en Ecuador, quien encontró un $15 \%$ de ISO. No obstante; en un hospital del Perú del mismo nivel resolutivo la incidencia de ISO $(41,9 \%)$ fue mayor que la reportada por nuestro estudio ${ }^{(5)}$.

El grado de infección de sitio operatorio que predominó en el estudio fue el grado 1 (incisional superficial), al igual que en el estudio de Rodríguez Fernández ${ }^{(12)}$, que encuentra una frecuencia de $21,1 \%$.

El diagnóstico postoperatorio hallado en la mayoría de los pacientes con ISO fue el de apendicitis aguda complicada, siendo la forma perforada la más frecuente siendo acorde a lo hallado por Hernández Romero ${ }^{(4)}(42 \%)$ y por Rodríguez Fernández ${ }^{(12)}(75,8 \%)$.

Con respecto a la asociación entre la técnica operatoria e infección de sitio operatorio, en nuestro estudio se pudo apreciar que, al realizar una apendicectomía abierta, se incrementaba el riesgo de sufrir una infección de sitio operatorio comparado con la apendicectomía laparoscópica, tal como se evidenció en el estudio de Guanche et al. ${ }^{(7)}$ (IRR: 2,3 IC95 \% 5,51-27,79) y en el estudio de Xiao et al. ${ }^{(13)}(4.5 \%$ vs $6.7 \%, \mathrm{P}<0.001)$.

En nuestro estudio, la mediana del tiempo de enfermedad no fue estadísticamente significativa, en comparación con el estudio de Rodríguez Fernández ${ }^{(12)}$ y Pérez et al. ${ }^{(14)}$ donde la mediana del tiempo de enfermedad fue mayor $(50,6 \mathrm{~h})$ en los casos que presentaron complicaciones $(p<0,01)$. Asimismo, no se encontró asociación entre ISO, sexo y edad ( $p=0,26$ y $p>0,99$, respectivamente) al igual que se evidenció en el estudio de Rocha-Almazán et al. (15) 
que tampoco hallaron diferencias significativas $(p=0,89)$.

En relación a las comorbilidades, la que predominó fue diabetes mellitus $(3,3 \%)$ pero, a pesar de ello, no mostró una significancia estadística ( $p>0,99)$. Lo mismo se puede observar en el estudio de Rocha-Almazán et al. (15), que encontró una prevalencia de $9 \%$ para diabetes mellitus y $12 \%$ para hipertensión arterial, sin embargo, ninguno de ellos se asoció a ISO $(p=0,63)$; al igual que el estudio de Guanche Garcell et al. (7) quienes reportaron una prevalencia de $6,3 \%$ de diabetes mellitus $(p>0,05)$.

Nuestro estudio evidencia una asociación entre la técnica operatoria de apendicectomía empleada y la presencia de ISO, sin embargo tiene la limitación de que los datos fueron obtenidos de los registros de las historias clínicas de una cohorte retrospectiva, no se pudieron explorar otras variables independientes de interés para los investigadores como adecuada técnica de lavado de mano, experticia del cirujano, comorbilidades, niveles de albumina sanguínea, conteo de linfocitos, uso de drogas inmunosupresoras entre otros factores. Sin embargo, debemos señalar que existen pocos estudios a nivel nacional sobre la infección de sitio operatorio y apendicectomía, por lo que este estudio transversal nos permite determinar la incidencia y explorar factores asociados a la presentación del ISO, lo que da la oportunidad de establecer hipótesis posteriores para futuros estudios analíticos, que determinen otros factores asociados.

\section{REFERENCIAS BIBLIOGRÁFICAS}

1. López Tagle D, Hernández Ferrer M, Saldivar Arias T, Sotolongo Hernández T, Valdés Dupeyrón O. Infección de la herida quirúrgica. Aspectos epidemiológicos. Rev Cub Med Mil. 2007 Abr-Jun; 36(2).

2. Santalla A, López-Criado MS, Ruiz MD, Fernández-Parra J, Gallo JL, Montoya F. Infección de la herida quirúrgica. Prevención y tratamiento. Clin Invest Ginstol Obstet. 2007 Sep; 34(5): 167-211.

3. World Health Organization. Global guidelines on the prevention of surgical site infection [libro electrónico]. 2016. Disponible en: https://www.who.int/gpsc/ssi-guidelines/en/

4. Hernández Romero JS. Infección quirúrgica en pacientes apendicectomizados, en el Servicio de Cirugía, Hospital del IESS Riobamba 2009-2010 [Tesis]. Riobamba: Escuela Superior Politécnica de Chimborazo. Facultad de Salud Pública; 2010.

5. Nicho Chávez C. Factores que se asocian a Infección de Sitio Operatorio en pacientes post operados por apendicectomía convencional en el Hospital Nacional Hipólito Unanue durante el periodo enero-julio del 2015 [Tesis]. Lima: Universidad Ricardo Palma. Facultad de Medicina Humana; 2016.

6. Caballero Arévalo AM, Ríos García RC, Ramírez López DC. Apendicectomía trans-umbilical. Abordaje quirúrgico mínimamente invasivo. Gac Med Bol. 2010; 33(1): 28-33.

7. Guanche Garcell H, Villanueva Arias A, Pancorbo Sandoval CA, Guilarte García E, Valle Gamboa ME, Bode Sado A, et al. Incidence and etiology of surgical site infections in

appendectomies: a 3 year prospective study. Oman Med J. 2017 Jan; 32(1): 31-5.

8. Aranda-Narváez JM, Prieto-Puga Arjona T, García-Albiach B, Montiel-Casado MC, González-Sánchez AJ, Sánchez-Pérez $B$, et al. Infección de sitio quirúrgico tras apendicectomía urgente: tasa global y tipo según la vía de abordaje (abierta/ laparoscópica). Enferm Infecc Microbiol Clin. 2014 Feb; 32(2): 67-136.

9. Suh YJ, Jeong SY, Park KJ, Park JG, Kang SB, Kim DW, et al. Comparison of surgical-site infection between open and laparoscopic appendectomy. J Korean Surg Soc. 2012 Jan; 82(1): 35-9.

10. Peralta Vargas CE, López HA, Díaz Gil JR, Rodríguez Montoya RM, Angulo Guzmán WR. Infección de sitio operatorio en apendicectomizados en el servicio de cirugía del Hospital III ESSALUD - Chimbote. Rev Gastroenterol Perú. 2004 Ene-Mar; 24(1): 43-9.

11. Ministerio de Salud. Compendio estadístico 2017 [libro electrónico]. Lima: Hospital María Auxiliadora. Disponible en: http://www.hma.gob.pe/pdf/publicaciones/29.pdf

12. Rodríguez Fernández Z. Complicaciones de la apendicectomía por apendicitis aguda. Rev Cubana Cir. 2010 Abr-Jun; 49(2).

13. Xiao Y, Shi G, Zhang J, Cao JG, Liu LJ, Chen TH, et al. Surgical site infection after laparoscopic and open appendectomy: a multicenter large consecutive cohort study. Surg Endosc. 2015 Jun; 29(6): 1384-93.

14. Pérez N, Romero M, Castelblanco MI, Rodríguez El. Infección del sitio operatorio de apendicectomías en un hospital de la orinoquia colombiana. Rev Colomb Cir. 2009; 24(1): 23-30.

15. Rocha-Almazán $M$, Sánchez-Aguilar $M$, Belmares-Taboada J, Esmer-Sánchez D, Tapia-Pérez JH, Gordillo-Moscoso A. Infección del sitio operatorio en cirugía abdominal no traumática. Cir Cir. 2008; 76: 127-31.

Fuentes de financiamiento:

Este artículo ha sido financiado por los autores.

Conflictos de interés:

Los autores declaran no tener ningún conflicto de interés.

\section{Correspondencia:}

Ysabel Lozano Rodas

Dirección: Av. Miguel Iglesias 968. San Juan de Miraflores. Lima, Perú. Teléfono: 974636541

Correo electrónico: onisa2012@hotmail.com

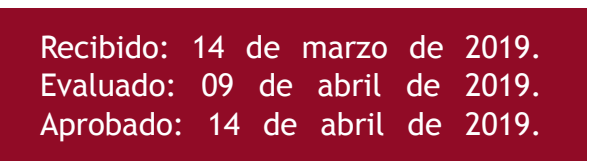

(c) La revista. Publicado por Universidad de San Martín de Porres, Perú. (c) вy Licencia de Creative Commons Artículo en acceso abierto bajo términos de Licencia Creative Commons Atribución 4.0 Internacional. (http://creativecommons.org/licenses/by/4.0/)

\section{ORCID iDs}

Ursula Alexandra Sandoval Giont Ysabel Lozano Rodas

Enver Vantroi Palacios Ordoñez

Jorge Antonio Kohatsu Yshida https://orcid.org/0000-0001-5281-1495 https: //orcid.org/0000-0002-9723-975X https://orcid.org/0000-0002-0832-8751 https://orcid.org/0000-0002-5617-948X 